\title{
A General Approach for All-visible-light Switching of Diarylethenes through Triplet Sensitization using Semiconducting Nanocrystals
}

Lili Hou ( $\boldsymbol{\sim}$ lilihou@tju.edu.cn )

Tianjin University https://orcid.org/0000-0001-9453-4924

\section{Wera Larsson}

Chalmers University of Technology https://orcid.org/0000-0003-1935-9884

\section{Stefan Hecht}

DWI - Leibniz Institute for Interactive Materials https://orcid.org/0000-0002-6124-0222

Joakim Andreasson

Chalmers University of Technology https://orcid.org/0000-0003-4695-7943

\section{Bo Albinsson}

Chalmers University of Technology https://orcid.org/0000-0002-5991-7863

\section{Article}

Keywords:

Posted Date: December 13th, 2021

DOI: https://doi.org/10.21203/rs.3.rs-1148200/v1

License: (c) (i) This work is licensed under a Creative Commons Attribution 4.0 International License. Read Full License 
1 A General Approach for All-visible-light Switching of Diarylethenes through

2 Triplet Sensitization using Semiconducting Nanocrystals

3 Lili Hou ${ }^{1,2,3 *}$, Wera Larsson ${ }^{1}$, Stefan Hecht ${ }^{4,5}$, Joakim Andréasson ${ }^{1}$, Bo Albinsson ${ }^{1 *}$

$4{ }^{1}$ Department of Chemistry and Chemical Engineering, Chalmers University of Technology,

5 Gothenburg 412 96, Sweden.

$6 \quad{ }^{2}$ Present Address: School of Precision Instruments and Optoelectronics Engineering, Tianjin

7 University, Tianjin 300072, China.

$8{ }^{3}$ Present Address: Key Laboratory of Optoelectronics Information Technology, Ministry of 9 Education, Tianjin 300072, China.

${ }^{4}$ Department of Chemistry \& IRIS Adlershof, Humboldt-Universität zu Berlin, Brook-TaylorStr. 2, 12489 Berlin, Germany.

${ }^{5}$ DWI-Leibniz Institute for Interactive Materials \& Institute of Technical and Macromolecular Chemistry at RWTH Aachen University, 52074 Aachen, Germany.

*Corresponding author e-mails: lilihou@tju.edu.cn; balb@chalmers.se

Abstract

Coupling semiconducting nanocrystals (NCs) with organic molecules provides an efficient route to generate and transfer triplet excitons. These excitons can be used to power photochemical transformations such as photoisomerization reactions using low energy radiation. Thus, it is desirable to develop a general approach that can efficiently be used to control photoswitches using all-visible-light aiming at future applications in life- and material sciences. Here, we demonstrate a simple 'cocktail' strategy that can achieve all-visible-light switchable diarylethenes (DAEs) through triplet energy transfer from the hybrid of CdS NCs and phenanthrene-3-carboxylic acid, with high photoisomerization efficiency and improved fatigue resistance. The size-tunable excitation energies of CdS NCs make it possible to precisely match the corresponding energy of the relevant DAE photoswitch. We demonstrate reversible all-visible-light photoisomerization of a series of DAE derivatives both in the liquid and solid state, even 

light activated optoelectronic devices as well as memories, and should in principle be adaptable to photopharmacology.

Introduction

Semiconducting nanocrystals (NCs), also known as quantum dots, are quantum confined inorganic crystals with high molar extinction coefficients often combined with stable and high photoluminescence (PL) quantum yields ${ }^{1}$. They have been used in a wide range of applications such as light-emitting devices, bioimaging, etc $^{2-4}$. The absorption and emission energies of NCs critically depend on their size, which can be precisely tuned by simply varying their synthesis conditions, in particular reaction temperature and time $\mathrm{e}^{5,6}$. Moreover, the strong spin-orbit coupling of NCs mixes the singlet and triplet characters and thereby enables the harvesting of triplet excitons from the NCs to surface-anchored molecules, referred to as mediators or transmitters. The hybrid of NCs and appropriate mediators yields a long triplet lifetime through highly efficient triplet energy transfer $(\mathrm{TET})^{7-9}$. This opens up further applications of $\mathrm{NCs} /$ molecule hybrids in high-performance light-emitting materials ${ }^{10}$ as well as solar energy converting devices through either singlet fission or triplet-triplet annihilation upconversion $(\text { TTA-UC) })^{11,12}$.

Triplet excited states are critical intermediates in many photochemical transformations including photocycloaddition and photoisomerization reactions. Coupling NCs with mediators provides an efficient route to power these photochemical transformations using low energy radiation. Weiss and co-workers recently demonstrated that CdSe NCs can catalyze regio- and diastereoselective intermolecular [2+2] cycloadditions ${ }^{13}$ and that $\mathrm{CuInS}_{2} / \mathrm{ZnS}$ NCs catalyze the photoreductive deprotection of aryl sulfonyl-protected phenols ${ }^{14}$. However, triplet sensitization 
from NCs to drive photoisomerization processes at longer and thus less harmful wavelengths remains a largely unexplored area.

Photoisomerization is the key photochemical process to operate photochromic molecular switches, often referred to as photoswitches, in which a structural change between two or more isomers is achieved by irradiation with light at different wavelengths ${ }^{15}$. Diarylethenes (DAEs) ${ }^{16,17}$, among the most extensively used molecular switches, have been widely applied as smart materials in constructing optical memories ${ }^{18}$, multi-responsive switchable devices ${ }^{19}$ as well as in bioimaging ${ }^{20}$ and photodynamic therapy ${ }^{21}$. Typically, ultraviolet (UV) light is required to induce efficient colorization via ring-closing isomerization (photocyclization) of DAEs. UV light causes photooxidation and thus degradation of the molecular switches and materials thereof. In addition, the penetration depth in tissue is very poor, which is a severe downside considering the potential use of these switches in photopharmacology $\mathrm{y}^{22,23}$. Many research efforts have been attributed to design all-visible-light activated DAEs ${ }^{24}$, such as shifting the absorption spectrum by chemically extending the $\pi$ conjugation $^{25,26}$, or using upconverting nanoparticles that absorb in the visible or near-infrared region to generate UV light to drive the photoisomerization ${ }^{27,28}$. However, directly shifting the absorption band of DAEs requires complicated synthesis, while the low efficiency of the upconverting process leads to overall low photoisomerization efficiencies. Triplet energy transfer from molecular triplet sensitizers has been proven to be another strategy $y^{29-33}$, however, the typical use of molecular sensitizers imposes limitations due to the rather constrained molecular design, relatively weak light absorption and high sensitivity towards oxygen. Thus, it is desirable to develop a general and improved approach that can be applied to a large set of DAEs using visible light only, while maintaining the high efficiency of photoisomerization and excellent fatigue resistance. 
77 PCA) to mediate the TET process, and it demonstrates a simple yet efficient noncovalent strategy to achieve photoisomerization of selected DAEs using light at different wavelengths in the visible region. The mechanism implies triplet-like excited states of the NCs lying only $\sim 20 \mathrm{meV}$ below the strong excitonic absorption band, which can sensitize the 'dark' triplet of the surface anchored mediator ${ }^{7,34}$. Subsequent TET from the mediator to the DAEs drives the photoisomerization along the triplet reaction pathway, as schematically illustrated in Figure 1a. The large absorption cross sections of CdS NCs enable unusually efficient visible light absorption compared to previously reported approaches using molecular sensitizers. The visible absorption band of CdS NCs can easily be tuned by varying the size of the CdS NCs to meet the transparent windows of the relevant DAE derivates, thereby driving the photoisomerization of diverse DAE photoswitches at desired visible wavelengths. The photoisomerization of our simple non-covalent 'cocktail' strategy upon visible light irradiation is as efficient as that of direct UV light irradiation and prevents photodegradation over multiple irradiation cycles. Moreover, the system can be operated both in solution and in the solid state. The switching of our systems can be retained at atmospheric environment, that is, it displays insensitivity to oxygen in the solid state, which is appealing in the fabrication of highperforming all-visible-light activated optoelectronics and memories. 
a

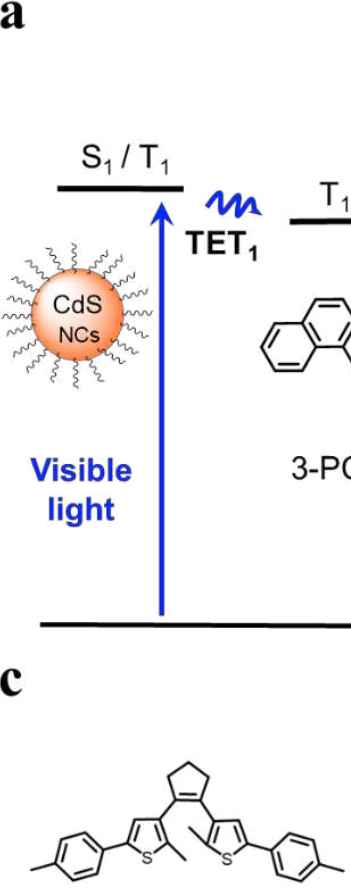

DAE1-open
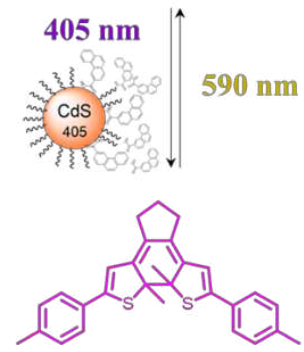

DAE1-closed

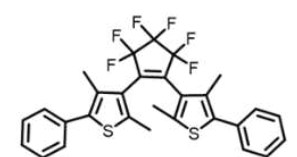

DAE2-open
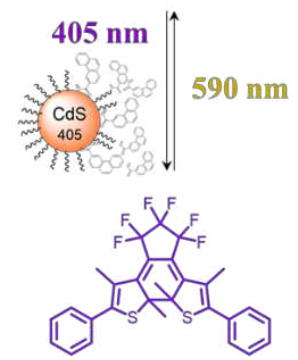

DAE2-closed

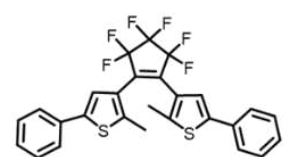

DAE3-open
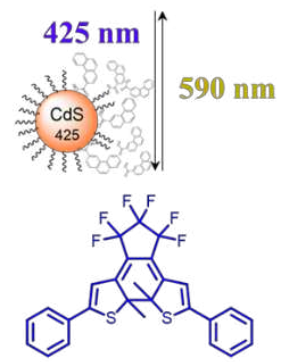

DAE3-closed b
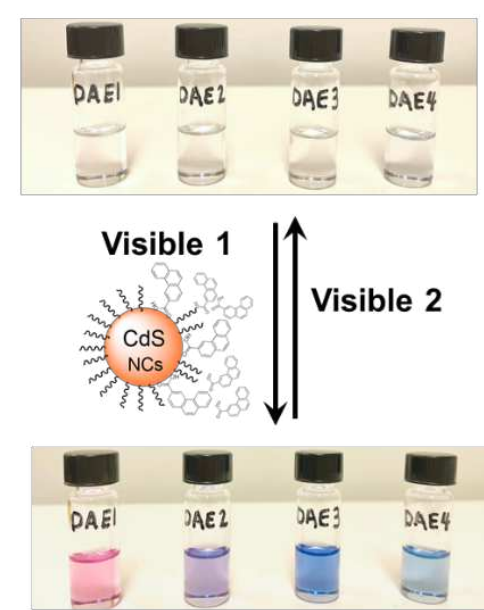

DAE4-open
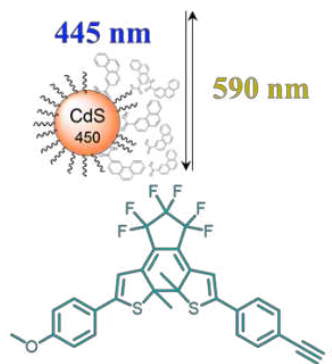

DAE4-closed

95

Figure 1. Schematic mechanism, color change and chemical structures of all-visible-light activated photoisomerization of DAEs through triplet energy transfer from CdS NCs and 3-PCA. a. Mechanism of the photoisomerization from DAE-open to DAE-closed under visible light irradiation via two triplet energy transfer steps $\left(\mathrm{TET}_{1}\right.$ and $\left.\mathrm{TET}_{2}\right)$, blue lines. The purple dashed lines indicate the direct UV-induced isomerization through the first singlet excited state.

b. Color changes of DAE derivatives under visible light irradiation in both isomerization directions in the presence of CdS NCs and 3-PCA. c. Chemical structures of DAEs. In view of the different absorption (color) of DAEs, CdS NCs with varied absorption bands are used to achieve irradiation at specific visible wavelengths. 
Materials and Mechanism. CdS NCs were synthesized according to previous reports ${ }^{35,36}$, see the details in the Methods/Synthesis section. The growth of CdS NCs during the reaction was monitored by measuring UV-visible absorption spectra of aliquots at different time intervals. Once the first absorption peak of CdS NCs reached the desired wavelength for the visible light irradiation study, the reaction was stopped by quickly cooling the solution to room temperature. Three batches of CdS NCs were prepared with the first absorption peak at $405 \mathrm{~nm}, 425 \mathrm{~nm}$, and $450 \mathrm{~nm}$, labeled $\mathrm{CdS} 405, \mathrm{CdS} 425$, and $\mathrm{CdS} 450$, respectively. Transmission electron microscopy (TEM) images, together with UV-visible absorption and PL spectra of the synthesized CdS NCs are shown in Supplementary Figure 1.3-PCA was synthesized to mediate the triplet energy of CdS NCs ${ }^{9}$. The carboxylic acid functional group enables the anchoring of 3-PCA to the surface of CdS NCs, which leads to efficient harvesting of long-lived 3-PCAlocalized triplets. Four DAE derivatives (Figure 1c) purchased or synthesized according to previous reports ${ }^{37-39}$ were used to investigate the photoswitching behavior upon visible light irradiation. PCA, as schematically indicated in Figure 1a. Visible light irradiation populates the excited state of the CdS NCs, from where the excitation energy is transferred to 3-PCA via the first step of triplet energy transfer $\left(\mathrm{TET}_{1}\right)$. The triplet energy of 3-PCA is then transferred to DAEopen via the second step of triplet energy transfer $\left(\mathrm{TET}_{2}\right)$, thereby sensitizing the photocyclization reaction along the triplet state pathway. Compared to directly driving the photocyclization of DAE through the singlet excited state upon UV light irradiation (the dashed purple path in Figure 1a), the energy transfer from the triplet state allows for the use of light at longer wavelength with all the advantages mentioned above. 
All-visible-light activated photoisomerization. To demonstrate the all-visible-light control, we first examined the photocyclization of a DAE derivative referred to as DAE1 (2-bis(2methyl-5-(p-tolyl)thiophen-3-yl)cyclopentene), in the presence of CdS 405 and 3-PCA upon visible light irradiation. Figure $2 \mathrm{a}$ shows the individual UV-visible absorption spectra of DAE1-open, DAE1-closed, CdS 405 and 3-PCA in toluene. DAE1-open displays no detectable absorption at wavelengths longer than around $350 \mathrm{~nm}$ (black spectrum in Figure 2a). Irradiation of DAE1-open at $302 \mathrm{~nm}$ (30 s, see details in Methods/Light sources) leads to the formation of DAE1-closed via the ring-closing isomerization, with the characteristic appearance of an absorption band in the visible region (red spectrum in Figure 2a). Concomitant change of the solution color from transparent to pink is observed. No spectral changes were observed for DAE1-open alone upon $405 \mathrm{~nm}$ visible light irradiation (Supplementary Figure 2). This is expected, as DAE1-open displays no detectable absorbance at $405 \mathrm{~nm}$ and it is also in agreement with the estimated energy of the singlet excited state $(4.2 \mathrm{eV})$ versus the photon energy at $405 \mathrm{~nm}(3.06 \mathrm{eV}) . \mathrm{CdS} 405$ was used as its first absorption band corresponds to a wavelength where DAE1-open and DAE1-closed have no or very low absorbance. Figure 2b shows UV-visible absorption spectra of the mixed solution of DAE1-open with CdS 405 and 3-PCA in deaerated toluene. The spectral features of DAE1 remain unchanged after mixing, indicating that there is no ground state interaction. Due to the high molar absorption coefficient of $\mathrm{CdS} 405, \varepsilon_{405}=4.0 \times 10^{5} \mathrm{M}^{-1} \mathrm{~cm}^{-1}$, only $0.5 \mu \mathrm{M}$ is required to initiate the photoisomerization of $50 \mu \mathrm{M}$ DAE1 under visible light exposure. Although the concentration of the mediator is $100 \mu \mathrm{M}$, it is completely transparent for wavelengths longer than $370 \mathrm{~nm}$, see the purple spectrum in Figure 2a. Upon visible light irradiation $(405 \mathrm{~nm}, 120 \mathrm{~s})$, the formation of DAE1closed follows as manifested by the appearance of an absorption band in the visible region (red spectrum in Figure 2b). The observed changes in the UV-visible absorption spectrum are identical to those observed for DAE1 alone upon direct UV light irradiation. Thus, the 
photoconversion of DAE1 in the presence of CdS 405 and 3-PCA upon exposure to $405 \mathrm{~nm}$ light yields a photostationary state (PSS) identical to that resulting from irradiation of DAE1 alone with $310 \mathrm{~nm}$ light, that is, $94 \%$ DAE1-closed ${ }^{40}$. The photocyclization quantum yield of DAE1 in the presence of CdS 405 and 3-PCA under $405 \mathrm{~nm}$ irradiation was determined to be $35 \%$, which is comparable to the quantum yield of DAE1 under direct $313 \mathrm{~nm}$ UV light irradiation $(43 \%)^{40}$.
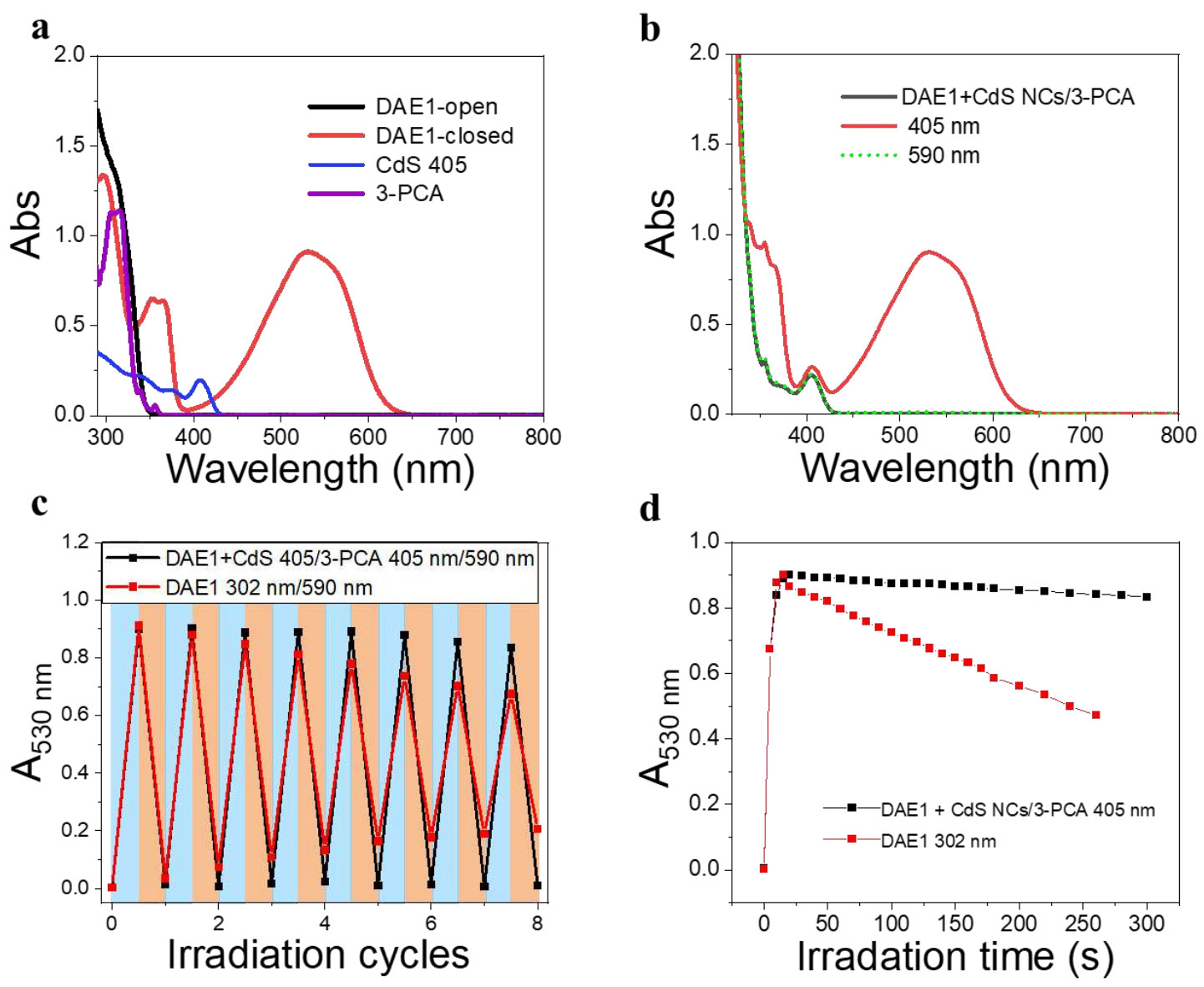

Figure 2. UV-visible absorption spectra of visible-light activated photoisomerization of

DAE1. a. UV-visible absorption spectra of the individual components: DAE1-open $(50 \mu \mathrm{M}$, black), DAE1-closed converted from the open form under $302 \mathrm{~nm}$ UV light irradiation (red), CdS $405(0.5 \mu \mathrm{M}$, blue $)$ and 3-PCA $(100 \mu \mathrm{M}$, purple) in toluene. b. UV-visible absorption spectra of a mixed solution of DAE1-open $(50 \mu \mathrm{M})$, CdS $405(0.5 \mu \mathrm{M})$, and 3-PCA $(100 \mu \mathrm{M})$ in deaerated toluene, before (black) and after light irradiation at $405 \mathrm{~nm}$ (120 s, red), and then 
after $590 \mathrm{~nm}$ light irradiation (90 s, green-dot). c. Reversibility of the photoswitching of the mixed solution as followed by the absorbance at $530 \mathrm{~nm}$ over eight irradiation cycles of 405 $\mathrm{nm}$ and $590 \mathrm{~nm}$ light (black), and DAE1 only over the irradiation cycles of $302 \mathrm{~nm}$ and 590 $\mathrm{nm}$ (red). Note that the maxima and minima truly correspond to the respective PSSs of forward and backward switching. d. Evolution of the $530 \mathrm{~nm}$ absorbance of DAE1-open in the mixture under $405 \mathrm{~nm}$ (black) and DAE1 only under $302 \mathrm{~nm}$ (red) irradiation over time.

Upon subsequent irradiation of the mixed solution with long-wavelength visible light $(590 \mathrm{~nm})$ for $90 \mathrm{~s}$, the band in the visible region disappears and the spectrum recovers to the initial state, see green-dot spectrum in Figure $2 b$. These observations confirm that when DAE1 is mixed with CdS 405 and 3-PCA, it can undergo reversible photoisomerization using visible light irradiation, that is, both the ring-opening and the ring-closing reactions are triggered without employing UV light. DAE1 shows fatigue over the switching cycles upon direct UV light irradiation, because of the formation of annulated ring byproducts ${ }^{41}$. Over eight irradiation cycles of alternating $302 \mathrm{~nm}$ light (30 s) and $590 \mathrm{~nm}(90 \mathrm{~s})$, nearly $50 \%$ degradation was observed (red in Figure 2c). The absorbance evolution also indicates that $\sim 50 \%$ DAE1 was degraded over 5 min of constant UV light irradiation (red in Figure 2d). In comparison, over eight irradiation cycles of alternating $405 \mathrm{~nm}(120 \mathrm{~s})$ and $590 \mathrm{~nm}$ (90 s) light exposure, the fatigue resistance of DAE1 is improved (black in Figure 2c), which is also evidenced under long time irradiation experiments (black in Figure 2d). The photoswitching behavior mentioned above indicates that triplet sensitization from $\mathrm{CdS}$ NCs and 3-PCA not only provides a strategy to achieve reversible all-visible-light activated DAEs, but can substantially improve the resistance to fatigue as the byproduct is generated mainly on the singlet surface ${ }^{40}$.

Mechanistic investigation of the triplet state sensitization. As the excited state lifetime of CdS NCs is too short (with an amplitude-weighted average lifetime of $\sim 16 \mathrm{~ns}$, Supplementary Section 3) to directly sensitize the triplet state of DAEs, a mediator that can anchor to the 
surface of CdS NCs with a long-lived triplet state (on the microsecond or millisecond timescale) is required. The triplet energy of the mediator should be located in between those of CdS NCs and DAE-open. The triplet-like state of CdS 405 is located at about $3.0 \mathrm{eV}$. The triplet state energies of DAE1-open and DAE1-closed are reported to be $2.5 \mathrm{eV}$ and $0.7 \mathrm{eV}$, respectively ${ }^{40}$. 3-PCA with a triplet state energy $(2.6 \mathrm{eV})$ close to DAE1-open was chosen as the mediator?. The efficiency of $\mathrm{TET}_{1}$ from CdS 405 to 3-PCA was determined to be close to unity $(91 \%$, see Supplementary Section 3). In the absence of 3-PCA as the mediator, $405 \mathrm{~nm}$ light irradiation did not result in any detectable ring-closing isomerization in a solution of DAE1 and CdS 405 (Supplementary Figure 4a). When using phenanthrene as the mediator for CdS 405, no isomerization of DAE1 under visible light irradiation was observed (Supplementary Figure 4b), suggesting that the $\mathrm{TET}_{1}$ step only occurs when the carboxylic acid group enables anchoring of the mediator to the surface of CdS NCs, because of the short-range Dexter-type triplet energy $\operatorname{transfer}^{7}$. When a mediator with a triplet state much lower than that of DAE1-open, such as 1pyrenecarboxylic acid $\left(\mathrm{PyCOOH}, \mathrm{T}_{1}=2.0 \mathrm{eV}\right)^{42}$ was used, no ring-closing isomerization was observed upon $405 \mathrm{~nm}$ irradiation (Supplementary Figure 4c) even if efficient TET ${ }_{1}$ from CdS NCs to PyCOOH occurred (Supplementary Figure 5).

The TET process was further investigated by time-resolved transient absorption (TA) spectroscopy. Our recent work shows that the triplet state of 3-PCA has a broad positive absorption band between $450 \mathrm{~nm}$ and $650 \mathrm{~nm}^{9} .415 \mathrm{~nm}$ pulsed laser excitation (2.0 mJ, $\left.10 \mathrm{~ns}\right)$ of CdS 405 mixed with an excess of 3-PCA induced the formation of triplet 3-PCA via the $\mathrm{TET}_{1}$ step, evidenced by the initial rise in the TA signal at $470 \mathrm{~nm}$ (black line in Figure $3 \mathrm{a}$ ). The subsequent decay of triplet 3-PCA corresponds to a lifetime of $22 \mu \mathrm{s}$, consistent with our previous observation ${ }^{9}$. Adding DAE1 to the CdS 405/3-PCA solution results in a decrease in the triplet lifetime of 3-PCA, due to the $\mathrm{TET}_{2}$ step from 3-PCA to DAE1-open. Figure $3 \mathrm{~b}$ shows an analysis by the Stern-Volmer equation, $\tau_{0} / \tau=1+\tau_{0} k_{q}[Q]$, where $\tau_{0}$ and $\tau$ are the triplet 
lifetimes of 3-PCA without and with DAE1, respectively, $k_{q}$ is the bimolecular quenching rate constant, and $[Q]$ is the concentration of the quencher, i.e. DAE1-open. The linear fit of the Stern-Volmer plot in Figure $3 \mathrm{~b}$ gives $k_{q}=1.4 \times 10^{9} \mathrm{M}^{-1} S^{-1}$, which is expected for an efficient diffusion-controlled process. Thus, the TA study demonstrates that the triplet states of 3-PCA formed in the TET 1 step are effectively transferred to DAE1 in the diffusion-controlled $\mathrm{TET}_{2}$ step.
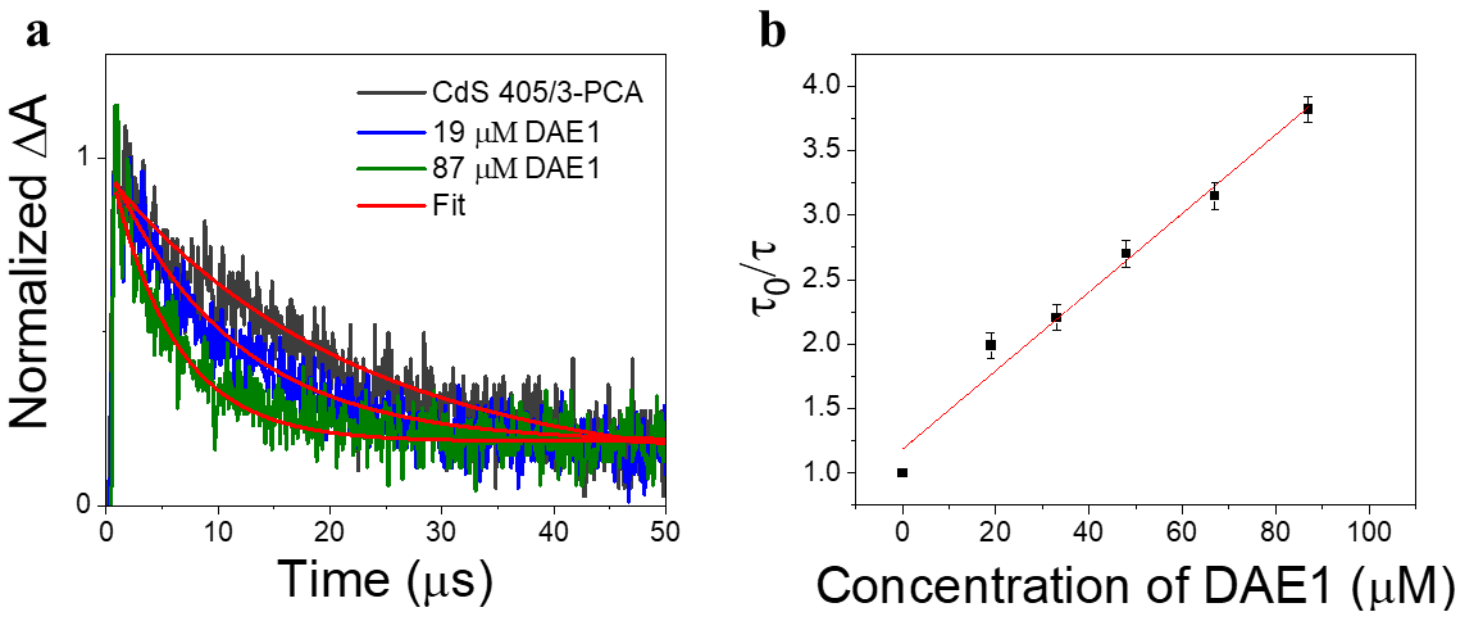

Figure 3. Quenching the triplet state of the CdS 405/3-PCA hybrid by adding DAE1. a.

Time-resolved transient absorption kinetics at $470 \mathrm{~nm}$ upon $415 \mathrm{~nm}$ pulsed excitation of CdS 405/3-PCA, and CdS 405/3-PCA mixed with DAE1-open in deaerated toluene. b. SternVolmer plot and linear fitting for triplet lifetime quenching of CdS 405/3-PCA by DAE1.

General application to DAE derivates. Our simple non-covalent cocktail strategy allows the hybrid of CdS NCs and 3-PCA to be used as a general triplet sensitizer for a variety of DAE derivatives under all-visible-light control. To demonstrate the general applicability, we further tested visible-light-activated photoswitching of a commercially available DAE derivative, 1,2bis(2,4-dimethyl-5-phenyl-3-thienyl)-3,3,4,4,5,5-hexafluoro-1-cyclopentene (DAE2). The sample was conveniently prepared by adding DAE2 into the solution of CdS 405 and 3-PCA in accordance with the procedure for DAE1. Figure 4a shows the UV-visible absorption spectra 
of the mixed solution and its response to visible light exposure. Upon $405 \mathrm{~nm}$ irradiation, DAE2 undergoes photoisomerization from the open form to the closed form, evidenced by the formation of the absorption band in the visible region and purple colorization of the sample, see the red spectrum in Figure 4a and the photo in Figure 1b. The photoconversion of DAE2 in the presence of CdS 405 and 3-PCA upon exposure to $405 \mathrm{~nm}$ light yields a PSS that contains $58 \%$ DAE2-closed, which is lower than that of direct UV irradiation at $313 \mathrm{~nm}(79 \%)^{43}$. We assign this observation to the relatively large spectral overlap between DAE2-closed and the excitation light at $405 \mathrm{~nm}$, resulting in a direct ring-opening reaction on the singlet excited manifold. The photocyclization quantum yield of DAE2 in the presence of CdS 405 and 3-PCA under $405 \mathrm{~nm}$ irradiation was determined to be $39 \%$, which is comparable to the quantum yield of DAE2 under direct $313 \mathrm{~nm}$ UV light irradiation $(46 \%)^{43}$. Upon $590 \mathrm{~nm}$ irradiation, the visible band of DAE2-closed disappears and the spectrum completely recovers to the initial state. The all-visible-light activated photoswitching of DAE2 is fully reversible without obvious fatigue as shown in Figure 4b. From density functional theory (DFT) calculations, the $\mathrm{T}_{1}$ state of DAE2-open is estimated to be $2.8 \mathrm{eV}$ (Supplementary Table 1), about $0.2 \mathrm{eV}$ higher than that of 3-PCA. A triplet energy transfer reaction will occur even under slightly endergonic conditions as described by the Sandros equation ${ }^{44}$ albeit with a slower rate. Triplet quenching experiments of the CdS 405/3-PCA hybrid by DAE2 and Stern-Volmer plot yield $k_{q}=2.7 \times$ $10^{7} \mathrm{M}^{-1} \mathrm{~s}^{-1}$ (See Figures $4 \mathrm{c}$ and $4 \mathrm{~d}$ ), which is almost one order of magnitude smaller than that observed for DAE1, in accordance with a less effective $\mathrm{TET}_{2}$ step due to endergonic triplet energy transfer. 

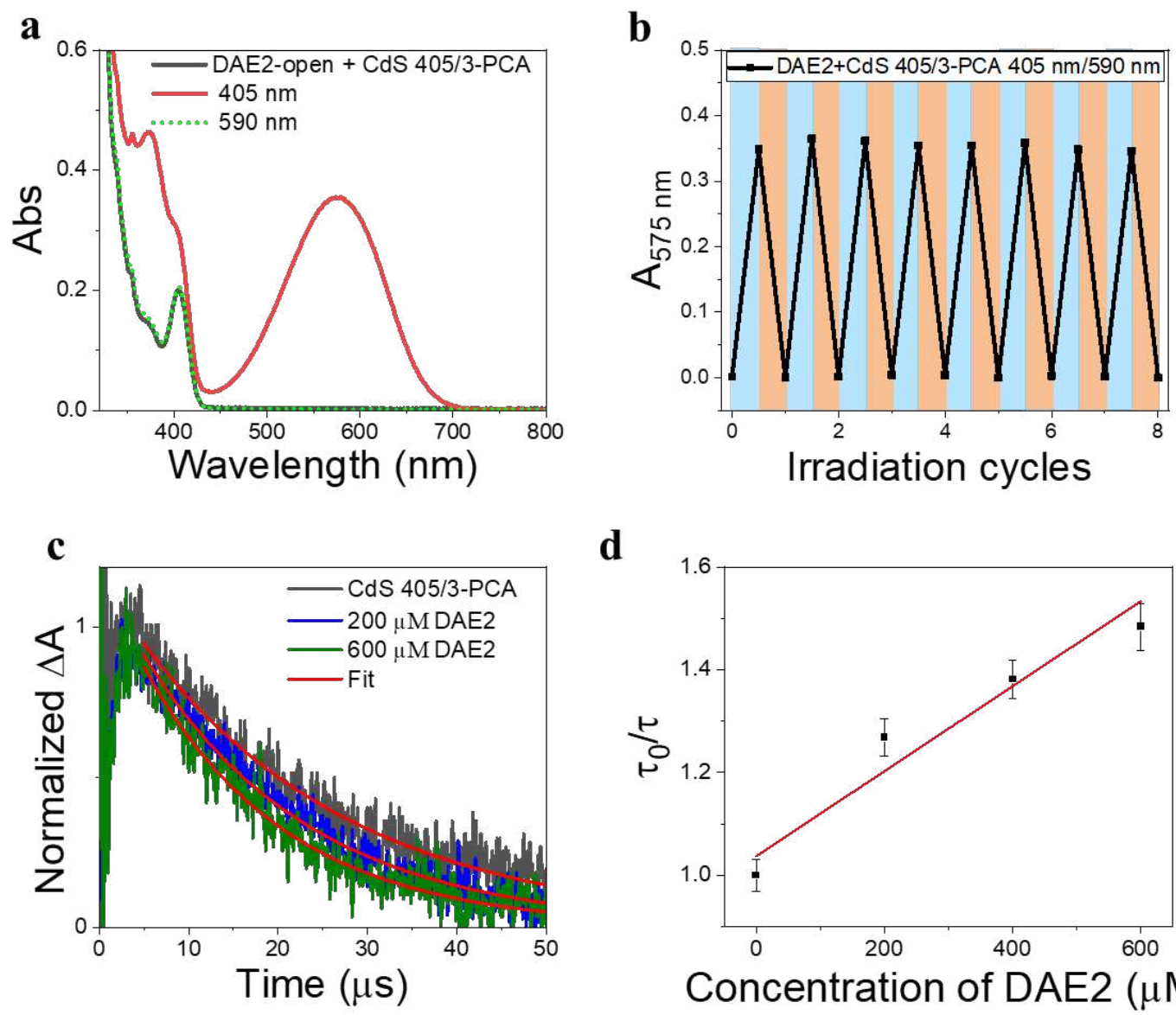

d

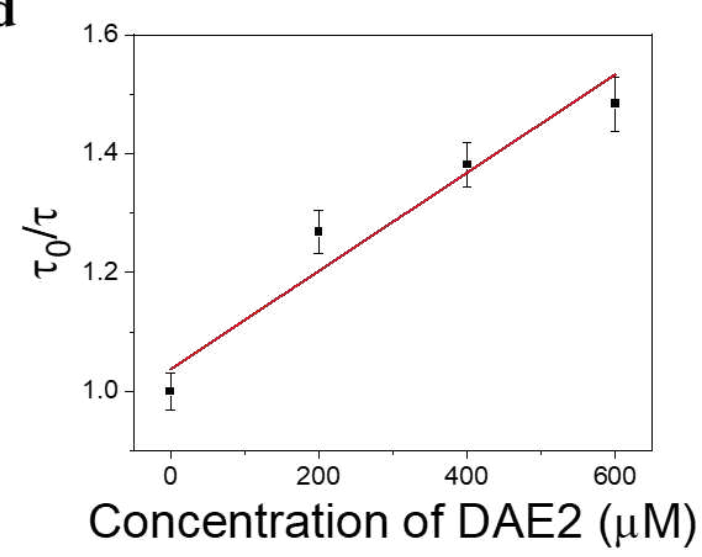

with DAE2. a. UV-visible absorption spectra of the solution of DAE2-open ( $50 \mu \mathrm{M}), \mathrm{CdS} 405$ $(0.5 \mu \mathrm{M})$, and 3-PCA $(100 \mu \mathrm{M})$ in deaerated toluene, before and after light irradiation at 405 $\mathrm{nm}$ (black and red) and then after $590 \mathrm{~nm}$ light irradiation (green-dot). b. Reversibility of the photoswitching as followed by the absorbance at $575 \mathrm{~nm}$ over eight irradiation cycles of 405 $\mathrm{nm}$ and $590 \mathrm{~nm}$ light. c. Time-resolved transient absorption kinetics at $450 \mathrm{~nm}$ upon $410 \mathrm{~nm}$ pulsed excitation of CdS 405/3-PCA and mixed with DAE2 in deaerated toluene. d. SternVolmer plot for triplet lifetime quenching of CdS 405/3-PCA by DAE2. photoisomerization also of other DAE derivatives in the desired visible wavelength window where both the ring-open and closed form are more or less transparent. As a demonstration, herein we used two other DAE molecules, referred to as DAE3 and DAE4, respectively. The 
wavelength window for these two derivatives allows for convenient excitation at $425 \mathrm{~nm}$ and $445 \mathrm{~nm}$, respectively, as seen in Figures 5a and 5c. Direct irradiation at $425 \mathrm{~nm}$ and $445 \mathrm{~nm}$ of the samples containing DAE3-open alone and DAE4-open alone, respectively, did not result in any ring-closing photoisomerization (see Supplementary Figure 6). CdS 425 and CdS 450 were synthesized to meet the absorption at the above-mentioned wavelengths (blue spectra in Figures 5a and 5c), and were used for the purpose of all-visible-light switching of DAE3 and DAE4. Figure 5b shows UV-visible absorption spectra of DAE3 mixed with CdS 425 and 3PCA in deaerated toluene. Upon $425 \mathrm{~nm}$ visible light irradiation, the formation of DAE3-closed was evidenced by the appearance of the typical visible absorption band and the color of the solution changed to blue, see the red spectrum in Figure 5b and the photo in Figure 1b. Figure $5 \mathrm{~d}$ shows the corresponding spectra of DAE4 mixed with CdS 450 and 3-PCA. Indeed, irradiation at $445 \mathrm{~nm}$ clearly triggers the photocyclization reaction for DAE4, as shown again by the appearance of the absorption band in the visible region and the solution color changed to cyan, see the red spectrum in Figure $5 \mathrm{~d}$ and the photo in Figure 1b. After subsequent irradiation at $590 \mathrm{~nm}$ of the mixed solutions, the visible bands of DAE3 and DAE4 disappeared, and the spectra recovered to their initial states (green-dot spectra in Figures $5 \mathrm{~b}$ and $5 \mathrm{~d}$ ). The above-mentioned observations illustrate that the ring-closing photoisomerization of a variety of DAE derivatives can be activated at desired visible wavelengths by simply varying the sizes of CdS NCs, and the all-visible-light photoswitching processes are fully reversible. 
a

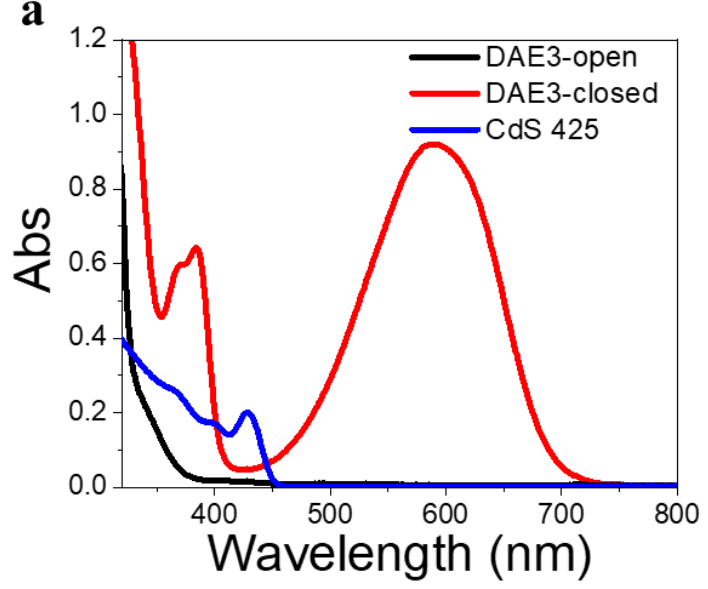

c

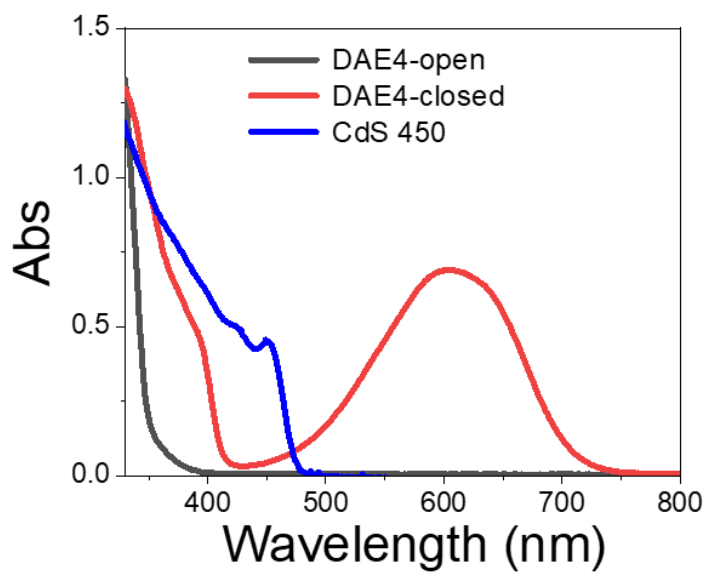

b

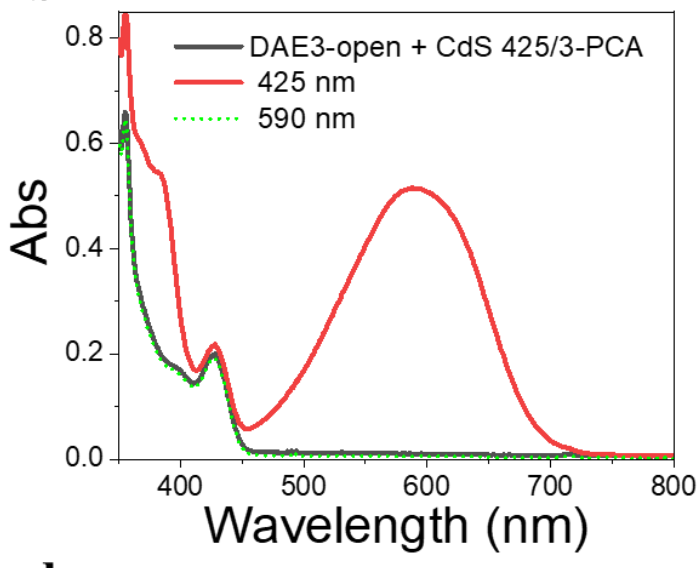

d

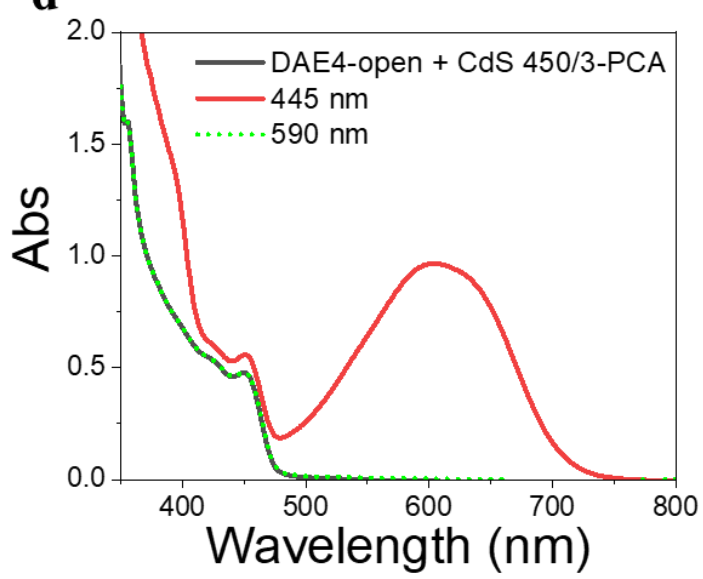

Figure 5. UV-visible absorption spectra of two DAE derivatives under tunable visible

irradiation. a. Individual UV-visible absorption spectra of DAE3-open (50 $\mu \mathrm{M}$, black),

DAE3-closed (formed under UV light irradiation, red), and CdS $425(0.3 \mu \mathrm{M}$, blue) in toluene.

b. UV-visible absorption spectra of the mixed solution of DAE3-open, CdS 425, and 3-PCA in deaerated toluene, before and after irradiation at $425 \mathrm{~nm}$ (pulsed laser, $10 \mathrm{~min}$ ) and then after $590 \mathrm{~nm}$ light irradiation (60 s). c. Individual UV-visible absorption spectra of DAE4-open (50 $\mu \mathrm{M}$, black), DAE4-closed (formed under UV light irradiation, red), and CdS $450(0.5 \mu \mathrm{M}$, blue) in toluene. d. UV-visible absorption spectra of the mixed solution of DAE4-open, CdS 450, and 3-PCA in deaerated toluene, before and after light irradiation at $445 \mathrm{~nm}$ (continuous laser, $9 \mathrm{~min})$ and then after $590 \mathrm{~nm}$ light irradiation (60 s). 
299 All-visible-light switching in the solid state. Our excitation strategy also performs well in the 300 solid state. A solid sample was prepared by soaking a piece of filter paper in a solution containing CdS 405, 3-PCA, and commercially available DAE2, with the same concentrations that were used for the UV-visible absorption study. The solvent slowly evaporated at $60{ }^{\circ} \mathrm{C}$ in the dark. Different patterns can be generated and erased reversibly on this solid sample as demonstrated in Figure 6. Initially, the dry sample paper is white, and $405 \mathrm{~nm}$ light irradiation stimulates a white-to-purple color change of the entire paper. Subsequent irradiation with visible light at $590 \mathrm{~nm}$ fully reverses the color change. Moreover, the patterns of ' $\mathrm{L}$ ' and ' $\mathrm{H}$ ' shapes can be written and erased consecutively using masks on the same paper by simply altering between the two visible wavelengths. Although the TET reactions require diffusion to occur, which is limited in the solid state, we hypothesize that the slow evaporation of the solvent can cause the mediator 3-PCA to aggregate around the surface of CdS NCs. This would lead to a local concentration enhancement of the molecules, and the shorter intermolecular distances that follow would still allow for efficient TET reactions to occur, even in the presence of oxygen. We also observed that all-visible-light activated photoisomerization was possible in an air-equilibrated solution (Supplementary Figure 7), although the photocyclization efficiency was a factor of 3-5 times lower than that in the deaerated case. Thus, our strategy of combining CdS NCs, 3-PCA and DAEs in the solid state can be used to generate different patterns reversibly and reproducibly, which holds great potential in all-visible-light responsive materials for optical memory and data storage. 


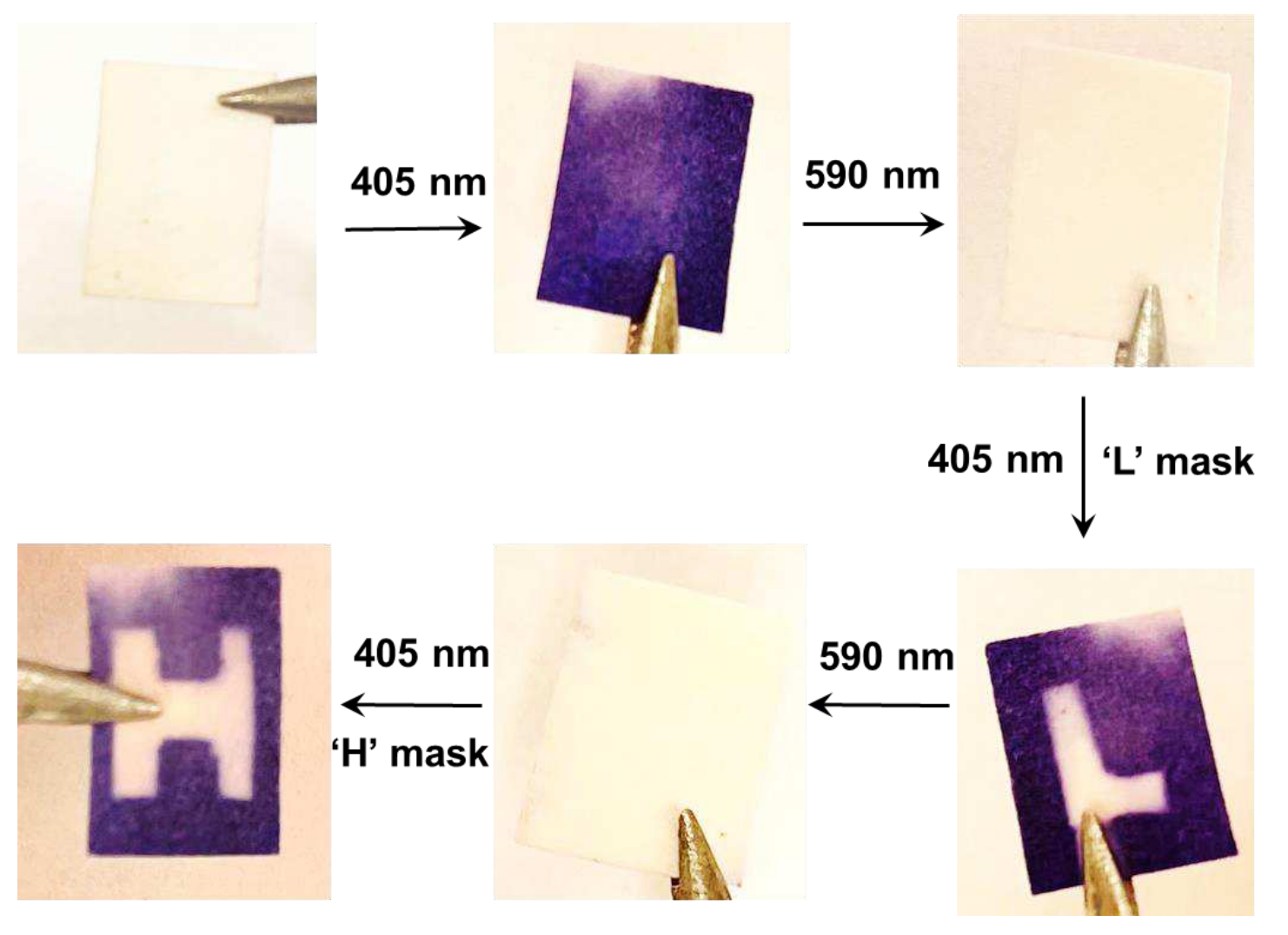

320 Figure 6. All-visible-light switching in the solid state. Images of a filter paper prepared from

321 CdS 405, 3-PCA and DAE2 solution, and its subsequent color (white and purple) and pattern

322 change ('L' shape and ' $H$ ' shape), upon visible light irradiation by simply varying the

323 wavelength between $405 \mathrm{~nm}$ and $590 \mathrm{~nm}$ in normal atmospheric environment.

324 Discussion

325 In summary, we have demonstrated that the hybrid of CdS NCs and 3-PCA can induce all-

326 visible-light activated photoisomerization of diarylethene photoswitches through triplet energy

327 transfer. This represents a novel strategy to achieve all-visible-light controlled photoswitchable

328 systems at selective wavelengths both in solution and in the solid state, even in the presence of

329 oxygen. Our systems are prepared by a 'cocktail' approach, that is, simply by mixing the

330 molecular building blocks and the NCs via non-covalent assembly. The quantum yield of

331 photocyclization through triplet energy transfer from CdS NCs/3-PCA is almost as high as that

332 of direct UV light irradiation, and the hybrid system displays better fatigue resistance over 
multiple irradiation cycles. NCs are par excellence candidates for this approach due to their appealing photophysical properties (high molar absorptivity, small singlet-triplet energy gap, and near unity efficiency of triplet energy transfer). It should be stressed that our strategy is generally applicable to a large variety of DAE derivatives with different absorption spectra and energy levels, due to the ability to precisely adjust the absorption spectra of the NCs by size variations. Further development of DAE derivatives with carboxylic acid functional groups can simplify the current three-component system to a bi-component system by excluding the mediator. Our simple yet effective non-covalent assembly approach paves the way to convenient fabrication of all-visible-light controlled photoswitchable optoelectronic devices and memories. Moreover, in the case of using non-toxic NCs, it should allow to non-invasively address bioactive switches in the context of photopharmocology.

\section{Data availability}

The data that support the findings of this study are available from the corresponding authors upon reasonable request.

\section{Methods}

\section{Chemicals}

Analytical reagent grade toluene from VWR was used without further purification for spectroscopic measurements. 1,2-bis(2,4-dimethyl-5-phenyl-3-thienyl)-3,3,4,4,5,5-hexafluoro-1-cyclopentene (DAE2) and 1-pyrenecarboxylic acid (PyCOOH) were purchased from TCI. Phenanthrene was purchased from Fluka A.G. The detailed synthesis of 3-PCA, DAE1, DAE3 and DAE4 was according to previous reports ${ }^{9,37-39}$. CdS NCs were prepared by a slightly modified synthesis according to previous reports $^{35,36}$.

Cadmium oxide, sulfur powder, 1-octadecene, and dioxane were purchased from Sigma Aldrich. Oleic acid was purchased from Alfa Aesar. 3-acetylphenanthrene was purchased from Acros Organics, 
sodium hypochlorite solution was purchased from Scharlau Chemie, and diethyl ether was purchased from Fisher Chemicals. All the chemicals were used without further purification steps.

\section{Synthesis}

CdS NCs: $257 \mathrm{mg}$ of CdO, $6 \mathrm{ml}$ of oleic acid and $15.8 \mathrm{ml}$ of ODE were mixed in a $50 \mathrm{ml}$ three-necked

flask, which was degassed under vacuum at $110{ }^{\circ} \mathrm{C}$ for 1 hour. After that, the solution was heated to $260{ }^{\circ} \mathrm{C}$ under $\mathrm{N}_{2}$ until the mixture turned colorless and clear. $32 \mathrm{mg}$ of sulfur was dissolved in $3 \mathrm{ml}$ of ODE, and degassed under $\mathrm{N}_{2}$ for 30 minutes, followed by sonication for at least 1 hour. The sulfur solution was injected into the $\mathrm{Cd}$ solution at $210^{\circ} \mathrm{C}$. After injection, the solutions were cooled by compressed air to $150-190{ }^{\circ} \mathrm{C}$ according to the size required. UV-visible absorption spectra of the aliquots were measured during the reaction. Once the first absorption peak of CdS NCs reached the desired wavelength, the reaction was stopped by fast cooling to room temperature. The obtained CdS NCs were further purified by extraction with hexane/acetonitrile three times and precipitated with acetone twice.

\section{Spectroscopies}

UV-visible absorption spectra were measured using a Cary 50 UV-vis-NIR spectrophotometer. Steadystate fluorescence spectra were recorded on a Cary Eclipse fluorescence spectrophotometer. Fluorescence lifetime measurements were carried out using time-correlated single photon counting (TCSPC), which was excited by a $405 \mathrm{~nm}$ laser diode (PicoQuant) and recorded by an MCP-PMT detector (10 000 counts, 2048 channels). Time-resolved transient absorption measurements were carried out on a home-built system, in which an Nd:YAG laser (10 ns, $10 \mathrm{~Hz}$, Spectra-Physics, Quanta-Ray) equipped with an OPO (Spectra-Physics, primoScan) was used as a pump beam, and a quartz-halogen lamp was used as a probe light. The time-resolved decays were measured on a 9 stage PMT (Applied Photophysics) coupled with a monochromator (Oriel Cornerstone 130, Newport), and the signals were recorded on an oscilloscope (TDS 2022, Tektronix) communicated with a computer. Transmission electron microscopy (TEM) images were recorded on a Titan 80-300 TEM (FEI Co.) equipped with a 
monochromator, Cs probe-corrector, and Gatan image filter. The acceleration voltage of the TEM was $300 \mathrm{kV}$.

\section{Sample preparations}

All photophysical measurements were carried out in toluene using a $10 \mathrm{~mm}$ path quartz cuvette. The samples for UV-visible absorption measurements were prepared by purging with argon for at least 15 minutes. The samples for quantum yield and transient absorption measurements were prepared by at least 4 freeze-pump-thaw cycles. The solid sample was prepared by soaking a piece of filter paper into the solution for UV-visible absorption measurement, letting all the solvent slowly evaporate at $60{ }^{\circ} \mathrm{C}$ in the dark.

\section{Light sources}

$302 \mathrm{~nm}$ irradiation was performed using a UV analytic lamp ( $\sim 10 \mathrm{mWcm}^{-2}$ at the sample); $425 \mathrm{~nm}$ irradiation was carried out by using an Nd:YAG laser $(10 \mathrm{~ns}, 10 \mathrm{~Hz})$ equipped with an OPO set at 425 $\mathrm{nm}(2.0 \mathrm{~mJ} /$ pulse $) ; 405 \mathrm{~nm}$ irradiation $\left(\sim 60 \mathrm{mWcm}^{-2}\right)$ and $445 \mathrm{~nm}$ irradiation $\left(\sim 15 \mathrm{mWcm}^{-2}\right)$ were carried out by using continuous-wave lasers (Coherent, OBIS) coupled with a concave lens; $590 \mathrm{~nm}$ irradiation $\left(\sim 15 \mathrm{mWcm}^{-2}\right.$ at the sample) was carried out using an LED light source (LED Engin, FWHM $=20 \mathrm{~nm})$.

\section{Quantum yield and conversion of photocyclization reactions}

Photocyclization quantum yields were determined by using Ferrioxalate actinometry ( $405 \mathrm{~nm}, \Phi=1.14$ ) according to standard methods $\mathrm{s}^{45} .405 \mathrm{~nm}$ irradiation was carried out by using a continuous-wave laser (Coherent, OBIS) coupled with a neutral density (ND) filter to reduce the intensity.

The conversion of DAE1-open to DAE1-closed at the photostationary state (PSS) was reported as 94\% upon $310 \mathrm{~nm}$ irradiation ${ }^{40}$, and the molar absorption coefficient of DAE1-closed was determined to be $\varepsilon_{522}=1.97 \times 10^{4} \mathrm{M}^{-1} \mathrm{~cm}^{-1}$. The conversion of DAE2-open to DAE2-closed at the PSS was reported as $79 \%$ upon $313 \mathrm{~nm}$ irradiation ${ }^{43}$, according to which the molar absorption coefficient of DAE2-closed 
in toluene was determined to be $\varepsilon_{575}=1.25 \times 10^{4} \mathrm{M}^{-1} \mathrm{~cm}^{-1}$. The corresponding conversions at the PSS upon $405 \mathrm{~nm}$ light irradiation were calculated using the above molar absorption coefficients.

\section{Computational details}

Density functional calculations (DFT) were performed using the Gaussian 16 software package ${ }^{46}$ using the hybrid functional B3LYP and the basis set $6-311+\mathrm{G}(\mathrm{d}, \mathrm{p})$. Full optimization of the ground-state structure was followed by excited state calculations using the basis set 6-31G(d). Excited state energies (12 lowest of both triplet and singlet spin) were calculated using the time-dependent formalism (TDDFT).

\section{References}

1 Alivisatos, A. P. Semiconductor clusters, nanocrystals, and quantum dots. Science 271, 933937 (1996).

2 Sun, Q. et al. Bright, multicoloured light-emitting diodes based on quantum dots. Nat. Photonics 1, 717-722 (2007).

3 Michalet, X. et al. Quantum dots for live cells, in vivo imaging, and diagnostics. Science 307, 538-544 (2005).

4 Dai, X. L. et al. Solution-processed, high-performance light-emitting diodes based on quantum dots. Nature 515, 96-99 (2014).

5 Rossetti, R., Nakahara, S. \& Brus, L. E. Quantum Size Effects in the Redox Potentials, Resonance Raman-Spectra, and Electronic-Spectra of Cds Crystallites in Aqueous-Solution. J. Chem. Phys. 79, 1086-1088 (1983).

6 Wang, X., Zhuang, J., Peng, Q. \& Li, Y. D. A general strategy for nanocrystal synthesis. Nature 437, 121-124 (2005).

7 Mongin, C., Garakyaraghi, S., Razgoniaeva, N., Zamkov, M. \& Castellano, F. N. Direct observation of triplet energy transfer from semiconductor nanocrystals. Science 351, 369-372 (2016).

8 Thompson, N. J. et al. Energy harvesting of non-emissive triplet excitons in tetracene by emissive PbS nanocrystals. Nat. Mater. 13, 1039-1043 (2014).

9 Hou, L., Olesund, A., Thurakkal, S., Zhang, X. \& Albinsson, B. Efficient Visible-to-UV Photon Upconversion Systems Based on CdS Nanocrystals Modified with Triplet Energy Mediators. Adv. Funct. Mater. 31, 2106198 (2021).

10 Qin, C. J. et al. Triplet management for efficient perovskite light-emitting diodes. Nat. Photonics 14, 70-75 (2020).

11 Tabachnyk, M. et al. Resonant energy transfer of triplet excitons from pentacene to PbSe nanocrystals. Nat. Mater. 13, 1033-1038 (2014).

$12 \mathrm{Wu}, \mathrm{M}$. F. et al. Solid-state infrared-to-visible upconversion sensitized by colloidal nanocrystals. Nat. Photonics 10, 31-34 (2016).

13 Jiang, Y. S., Wang, C., Rogers, C. R., Kodaimati, M. S. \& Weiss, E. A. Regio- and diastereoselective intermolecular [2+2] cycloadditions photocatalysed by quantum dots. Nat. Chem. 11, 1034-1040 (2019).

14 Perez, K. A., Rogers, C. R. \& Weiss, E. A. Quantum Dot-Catalyzed Photoreductive Removal of Sulfonyl-Based Protecting Groups. Angew. Chem. Int. Ed. 59, 14091-14095 (2020). 
$44715 \quad$ Feringa, B. L. \& Browne, W. R. Molecular switches. 2nd edn, (Wiley-VCH, 2011).

44816 Tian, H. \& Yang, S. J. Recent progresses on diarylethene based photochromic switches. Chem. Soc. Rev. 33, 85-97 (2004).

17 Irie, M., Fulcaminato, T., Matsuda, K. \& Kobatake, S. Photochromism of Diarylethene Molecules and Crystals: Memories, Switches, and Actuators. Chem. Rev. 114, 12174-12277 (2014).

18 Leydecker, T. et al. Flexible non-volatile optical memory thin-film transistor device with over 256 distinct levels based on an organic bicomponent blend. Nat. Nanotechnol. 11, 769-775 (2016).

Hou, L. et al. Optically switchable organic light-emitting transistors. Nat. Nanotechnol. 14, 347353 (2019).

20 Zou, Y. et al. Amphiphilic Diarylethene as a Photoswitchable Probe for Imaging Living Cells. J. Am. Chem. Soc. 130, 15750-15751 (2008). of Singlet Oxygen Generation Using Diarylethene Photochromic Switches. J. Am. Chem. Soc. 136, 910-913 (2014).

22 Velema, W. A., Szymanski, W. \& Feringa, B. L. Photopharmacology: Beyond Proof of Principle. J. Am. Chem. Soc. 136, 2178-2191 (2014).

23 Broichhagen, J., Frank, J. A. \& Trauner, D. A Roadmap to Success in Photopharmacology. Accounts Chem. Res. 48, 1947-1960 (2015).

24 Bleger, D. \& Hecht, S. Visible-Light-Activated Molecular Switches. Angew. Chem. Int. Ed. 54, 11338-11349 (2015).

25 Tsivgoulis, G. M. \& Lehn, J. M. Multiplexing optical systems: Multicolor-bifluorescent-biredox photochromic mixtures. Adv. Mater. 9, 627-630 (1997).

26 Fukaminato, T. et al. Molecular Design Strategy toward Diarylethenes That Photoswitch with Visible Light. J. Am. Chem. Soc. 136, 17145-17154 (2014).

27 Boyer, J. C., Carling, C. J., Gates, B. D. \& Branda, N. R. Two-Way Photoswitching Using One Type of Near-Infrared Light, Upconverting Nanoparticles, and Changing Only the Light Intensity. J. Am. Chem. Soc. 132, 15766-15772 (2010).

28 Wu, T. \& Branda, N. R. Using low-energy near infrared light and upconverting nanoparticles to trigger photoreactions within supramolecular assemblies. Chem. Commun. 52, 8636-8644 (2016).

29 Jukes, R. T. F., Adamo, V., Hartl, F., Belser, P. \& De Cola, L. Photochromic dithienylethene derivatives containing Ru(II) or Os(II) metal units. Sensitized photocyclization from a triplet state. Inorg. Chem. 43, 2779-2792 (2004).

30 Yam, V. W. W., Ko, C. C. \& Zhu, N. Y. Photochromic and luminescence switching properties of a versatile diarylethene-containing 1,10-phenanthroline ligand and its rhenium(I) complex. J. Am. Chem. Soc. 126, 12734-12735 (2004).

31 Fredrich, S., Gostl, R., Herder, M., Grubert, L. \& Hecht, S. Switching Diarylethenes Reliably in Both Directions with Visible Light. Angew. Chem. Int. Ed. 55, 1208-1212 (2016).

32 Zhang, Z. W. et al. Diarylethenes with a Narrow Singlet-Triplet Energy Gap Sensitizer: a Simple Strategy for Efficient Visible-Light Photochromism. Adv. Opt. Mater. 6, 1700847 (2018).

33 Zhang, Z. W. et al. A building-block design for enhanced visible-light switching of diarylethenes. Nat. Commun. 10, 4232 (2019).

34 Efros, A. L. et al. Band-edge exciton in quantum dots of semiconductors with a degenerate valence band: Dark and bright exciton states. Phys. Rev. B 54, 4843-4856 (1996).

$35 \mathrm{Yu}, \mathrm{W}$. W. \& Peng, X. G. Formation of high-quality CdS and other II-VI semiconductor nanocrystals in noncoordinating solvents: Tunable reactivity of monomers. Angew. Chem. Int. Ed. 41, 2368-2371 (2002). 

with Elemental Sulfur Activation and Its Implication in Synthetic Development. J. Am. Chem. Soc. 133, 17248-17256 (2011).

37 Orgiu, E. et al. Optically switchable transistor via energy-level phototuning in a bicomponent organic semiconductor. Nat. Chem. 4, 675-679 (2012).

Liddell, P. A., Kodis, G., Moore, A. L., Moore, T. A. \& Gust, D. Photonic switching of

502

503

504 photoinduced electron transfer in a dithienylethene-porphyrin-fullerene triad molecule. J. Am. Chem. Soc. 124, 7668-7669 (2002).

505

506

507

508

509

510

511

512

39 Hermes, S. et al. New fast synthesis route for symmetric and asymmetric phenyl-substituted photochromic dithienylethenes bearing functional groups such as alcohols, carboxylic acids, or amines. Tetrahedron Lett. 50, 1614-1617 (2009).

40 Herder, M. et al. Improving the Fatigue Resistance of Diarylethene Switches. J. Am. Chem. Soc. 137, 2738-2747 (2015).

41 Irie, M., Lifka, T., Uchida, K., Kobatake, S. \& Shindo, Y. Fatigue resistant properties of photochromic dithienylethenes: by-product formation. Chem. Commun., 747-748 (1999).

42 Mongin, C., Moroz, P., Zamkov, M. \& Castellano, F. N. Thermally activated delayed photoluminescence from pyrenyl-functionalized CdSe quantum dots. Nat. Chem. 10, 225-230 (2018).

43 Irie, M., Sakemura, K., Okinaka, M. \& Uchida, K. Photochromism of dithienylethenes with electron-donating substituents. J. Org. Chem. 60, 8305-8309 (1995).

44 Sandos, K. Transfer of Triplet Energy in Fluid Solutions III. Reversible Energy Transfer. Acta Chem. Scand. 18, 2355-2374 (1964).

45 Montalti, M. \& Murov, S. L. Handbook of photochemistry. 3rd edn, (CRC/Taylor \& Francis, 2006).

46 Frisch, M. J. et al. Gaussian 16, Reversion C. 01e. (2016).

Acknowledgements

We thank Dr. Xiaoyan Zhang and Dr. Shameel Thurakkal for synthesis of 3-PCA, Jutta Schwarz for synthesis of DAE1, and Dr. Shiming Li for synthesis of DAE3 and DAE4. B.A. acknowledges support from the Swedish Energy Agency (contracts: 46526-1 and 36436-2).

\section{Author contributions}

L.H. conceived the ideas and designed the project. S.H. and J.A. provided the diarylethene samples. L.H. synthesized CdS NCs. L.H. and W.L. performed the spectroscopy measurements and the data analysis. B.A. performed theoretical calculations. All authors discussed the results and contributed to interpretation of data. L.H. wrote the manuscript with contributions from all authors.

\section{Competing interests}

531 The authors declare no competing interests.

\section{Correspondence}

Prof. Dr. Lili Hou ${ }^{1,2,3 *}$

534 orcid.org/0000-0001-9453-4924; Email: lilihou@tju.edu.cn

$535{ }^{1}$ Department of Chemistry and Chemical Engineering, Chalmers University of Technology, Gothenburg 536412 96, Sweden.

$537{ }^{2}$ Present Address: School of Precision Instruments and Optoelectronics Engineering, Tianjin University, 538 Tianjin 300072, China. 
$539{ }^{3}$ Present Address: Key Laboratory of Optoelectronics Information Technology, Ministry of Education, 540 Tianjin 300072, China.

541 Prof. Dr. Bo Albinsson ${ }^{1 *}$

542 orcid.org/0000-0002-5991-7863; E-mail: balb@chalmers.se

$543{ }^{1}$ Department of Chemistry and Chemical Engineering, Chalmers University of Technology, Gothenburg 54441296, Sweden. 


\section{Supplementary Files}

This is a list of supplementary files associated with this preprint. Click to download.

- SIAGeneralApproachforAllvisiblelightSwitchingofDAEsusingNCs.docx 